\title{
Efficient Assembly of Large Multiplex CRISPR/Cas9 Guide Arrays for Maize Genome Editing
}

Jarrett Man* and Madelaine E Bartlett

Biology Dept, University of Massachusetts Amherst, Amherst, MA 01003, USA

*For correspondence: jaman@umass.edu

[Abstract] CRISPR/Cas9-based genome editing in maize is an effective tool, and researchers commonly wish to target multiple genes simultaneously. Transformation of maize is currently expensive and tedious, so researchers are incentivized to build vectors that target multiple genomic loci from a single transformation event. One way to accomplish this is to arrange Cas9 guides into a multiplex array that is transformed as a single locus to the plant (Char et al., 2017). These arrays can be long and repetitive, are challenging to build with traditional assembly methods such as restriction cloning, and are also difficult and expensive to synthesize. Golden Gate gene assembly (Vad-Nielsen et al., 2016) is a good answer to this challenge, as it is insensitive to the tandem repeats in these arrays. The MoClo system is an elaboration of Golden Gate cloning (Werner et al., 2012) and is particularly well suited for assembling larger multiplexed Cas9 guide arrays. In this protocol, we describe steps for designing and building a custom guide array targeting any number of maize loci using the MoClo standard components and syntax. We provide instructions for using variations of the maize and rice U6 promoters to drive guide RNA expression, but this system is generalizable for constructing guide arrays for other species as well.

Keywords: CRISPR, Maize, Cas9, Array, Multiplex

[Background] Generating large arrays of guides is an efficient way to target multiple genomic loci from a single transformation event, which is especially relevant for species that are difficult to transform, like maize. These arrays have many small components and are repetitive, features that are difficult to achieve with traditional molecular assembly methods such as Gibson or restriction-ligation assembly, or newer gene synthesis methods. Golden Gate molecular assemblies utilize type IIS restriction endonucleases to guide the assembly of multiple DNA fragments into large constructs and is particularly well suited to building large Cas9 guide arrays. The MoClo system builds on Golden Gate cloning by utilizing a specialized syntax and set of vectors for the hierarchal iterative assembly of any number of DNA fragments in any arrangement (Werner et al., 2012). The MoClo system is therefore ideal for producing the large guide arrays that are an efficient way to edit multiple loci in difficult to transform systems such as maize.

Method overview: Here, we describe the process for Moclo assembly of a Cas9 guide gene array for gene editing in maize, using variants of maize and rice U6 promoters for driving guide RNA expression (Char et al., 2017; Qi et al., 2018). To accomplish this, the MoClo system is used in several steps to assemble small genetic parts into increasingly large and complex genetic constructs. In the Moclo language, three Level 0 parts are first produced: a promoter, a guide noticeable spacer, and an 
sgRNA scaffold. Next, these three Level 0 parts are assembled together to make a single Level 1 gene targeting a unique genomic locus. Multiple Level 1 genes with unique guide spacers are constructed in parallel, then assembled together to make a Level 2 multiplex array. Subsequent rounds of assembly can add more Level 1 genes to the array, with no theoretical limit to size. Finally, the array is transferred to an Agrobacterium expression vector to be used for maize transformation.

\section{Materials and Reagents}

1. $1,000 \mu \mathrm{l}, 200 \mu \mathrm{l}, 20 \mu \mathrm{l}$, and $2 \mu \mathrm{l}$ tips

2. $1.7 \mathrm{ml}$ Microcentrifuge tubes (Thermo Fisher Scientific, catalog number: 05-408-129)

3. Standard $90 \mathrm{~mm}$ Petri Dishes (Thermo Fisher Scientific, catalog number: 101VR20)

4. Agrobacterium tumefaciens strain EHA101 (Life Science Market, catalog number: STR3010) Note: Homemade preparations are also fine.

5. Chemically competent $E$. coli cells (One Shot ${ }^{\mathrm{TM}}$ TOP10 Chemically Competent $E$. coli) (Thermo Fisher Scientific, catalog number: C404010)

Note: Homemade preparations are also fine. Store for 6 months at $-80^{\circ} \mathrm{C}$.

6. Maize B73 genomic DNA, diluted to $50 \mathrm{ng} / \mathrm{\mu l}$

7. Rice Nipponbare genomic DNA, diluted to $50 \mathrm{ng} / \mathrm{\mu l}$

8. Ultrapure water

9. SOC media (Thermo Fisher Scientific, catalog number: 15544034)

Note: Homemade preparations are also fine. Store indefinitely at $-20^{\circ} \mathrm{C}$.

10. The MoClo Toolkit (Addgene, catalog number: 1000000044) is highly recommended, although the components can be purchased individually

Note: This is a 96-well plate of frozen glycerol stocks. Store indefinitely at $-80^{\circ} \mathrm{C}$.

11. Enzymes (generally last several years when stored at $-20^{\circ} \mathrm{C}$ )
a. T4 DNA ligase (New England BioLabs, catalog number: M0202S)
b. Bsal (Bsal-HF${ }^{\circledR}$ V2) (New England BioLabs, catalog number: R3733S)
c. Bpil (Thermo Fisher Scientific, catalog number: ER1011); Alternative Bpil Isoschizomer: Bbsl-HF (New England BioLabs, catalog number: R3539S)
d. Dpnl (New England BioLabs, catalog number: R0176S)

12. pENTR $^{\mathrm{TM}}$ Directional TOPO ${ }^{\circledR}$ Cloning Kit, including PENTR $^{\mathrm{TM}}$ cloning vector (Thermo Fisher Scientific, catalog number: K240020)

13. Gateway cloning kit (Gateway ${ }^{\mathrm{TM}}$ LR Clonase $^{\mathrm{TM}}$ II Enzyme mix, Invitrogen, catalog number: 11791100)

14. pMCG1005 Gateway cloning vector with Cas9 gene (lab stock)

15. Standard LB bacteria growing plates with the appropriate antibiotics for cloning

16. Antibiotics (will last several years when stored at $-20^{\circ} \mathrm{C}$ )
a. Spectinomycin (Sigma-Aldrich, catalog number: S0692)
b. Carbenicillin (Sigma-Aldrich, catalog number: C1613) 
c. Kanamycin (Sigma-Aldrich, catalog number: 60615)

d. Rifampicin (Sigma-Aldrich, catalog number: R3501)

17. High-fidelity DNA polymerase (Phusion ${ }^{\circledR}$ High-Fidelity DNA Polymerase) (New England BioLabs, catalog number: M0530S)

18. Agarose I (Thermo Fisher Scientific, catalog number: 17850)

19. Glycerol (Thermo Fisher Scientific, catalog number: 17904)

20. Commercial DNA cleaning column kit (QIAquick PCR Purification Kit) (QIAGEN, catalog number: 28106)

21. X-gal solution, ready-to-use (Thermo Fisher Scientific, catalog number: R0941)

22. IPTG (Thermo Fisher Scientific, catalog number: 34060)

23. Primers for amplification of promoters (Table 1 )

Table 1. Primer sequence for amplification of promoters. All primers are listed in the $5^{\prime}-3^{\prime}$ orientation, the Reverse primers are the reverse complement sequence of the 3 'end of the promoters. Both short and long versions of the U6 promoters can be used. The relative efficiency of each of these is unclear at this time. Different promoters are typically shuffled to drive guides. Note that entire Reverse primer is the reverse complement of the end of the promoter sequence, but that the four bases highlighted in orange will be used as overlaps in the Golden Gate assembly. Using these bases as the assembly overlap, rather than standard Moclo overlap sequences, is necessary to prevent assembly scars and ensure cellular transcription of guides begins at the intended nucleotide.

\begin{tabular}{|l|l|l|l|}
\hline Promoter variant & Length (bp) & Forward primer sequence & Reverse primer sequence \\
\hline ZmU6-C1_Short & 178 & 5'-CACGTGAGCTTGCGATGTC-3' & 5'-AAAACGGTTGTCGCAGCTG-3' \\
\hline ZmU6-C1_Long & 571 & $\begin{array}{l}\text { 5'-CTTTTGTTTCACTTATCAGTTTTCACC- } \\
\text { 3' }\end{array}$ & 5'-AAAACGGTTGTCGCAGCTG-3' \\
\hline ZmU6-C3-Short & 194 & 5'-CAAAAACATCCTCACAGGAAAG-3' & 5'-GGTATGGTTCTTCCGCTCTG-3' \\
\hline ZmU6-C3_Long & 588 & 5'-CATGGACAGGACACACTGC-3' & 5'-GGTATGGTTCTTCCGCTCTG-3' \\
\hline OsU6.1_short & 187 & 5'-TGGGCCGTAACGGAGGATAC-3' & 5'-AACACAAGCGGCAGCGC-3' \\
\hline OsU6.1_Long & 330 & 5'-AAGAACGAACTAAGCCGGACA-3' & 5'-AACACAAGCGGCAGCGC-3' \\
\hline OsU6.2_short & 163 & 5'-TGGGCTGGATGCATGCG-3' & 5'-AACACAAGCGACAGCGCG-3' \\
\hline OsU6.2_Long & 246 & 5'-GGATCATGAACCAACGGCCT-3' & 5'-AACACAAGCGACAGCGCG-3' \\
\hline
\end{tabular}

\section{Equipment}

1. $1,000 \mu \mathrm{l}, 200 \mu \mathrm{l}, 20 \mu \mathrm{l}$, and $2 \mu \mathrm{l}$ Pipettes 
2. Water bath (Precision ${ }^{\mathrm{TM}}$ General Purpose Baths) (Thermo Fisher Scientific, catalog number: TSGP02)

3. Tabletop centrifuge (Eppendorf ${ }^{\mathrm{TM}} 5424$ Microcentrifuge) (Fisher Scientific, catalog number: 05-403-93)

4. Spectrophotometer (NanoDrop ${ }^{\mathrm{TM}}$ One) (Thermo Scientific, catalog number: 13-400-519)

5. Thermocycler (Eppendorf ${ }^{\mathrm{TM}}$ Mastercycler ${ }^{\mathrm{TM}}$ pro) (Fisher Scientific, catalog number: E950030010)

6. Shaking incubator (Thermo Fisher Scientific, catalog number: 101VR20)

\section{Software}

1. NEBioCalculator tool (http://nebiocalculator.neb.com)

2. Genetic sequence analysis software such as Geneious (https://www.geneious.com) or Serial Cloner (http://serialbasics.free.fr/Serial Cloner.html)

\section{Procedure}

Procedure outline:

A. Design guides to target genomic loci

B. Build a custom Level 2 acceptor/Gateway entry clone

C. Build guide array parts and assemble into an array

D. Other general procedures (PCR, Golden Gate, bacterial transformation)

A. Design guides to target genomic loci

Note: Many excellent online tools are available for selecting targets. A good choice for maize is CRISPOR (Haeussler et al., 2016), which can work from the maize version 4 genome and allows filtering by several important criteria. It also contains many other species' genomes.

1. Consider genomic sequence between one quarter to halfway through of the gene's coding region. If required, input this sequence to the guide design tool.

2. If possible, target two nearby loci (ideally $<200 \mathrm{bp}$ apart). This can result in a dropout deletion between the guides (Rodríguez-Leal et al., 2017), inactivating the gene and facilitating genotyping.

3. The 20 bp guide sequence should have a " $G$ " at the $5^{\prime}$ most position when using the U6 promoters. If using the plant U3 promoter, the guide sequence should have " $\mathrm{A}$ " at this position. (Svitashev et al., 2015)

4. For this assembly method, guides should not contain either Bpil or Bsal restriction sites. Before proceeding, make an in-silico version of each guide with promoter and sgRNA scaffold to check if either Bpil or Bsal restriction sites are created. 
5. Mismatches causing off-targets are probably not a significant problem in maize (Feng et al., 2016; Lee et al., 2019), but it is still wise to reject any sequence with off-targets that have 2 or fewer mismatches whenever possible.

B. Build a custom Level 2 acceptor/Gateway entry clone

Note: Many expression vectors used for maize transformation, such as those based on pMCG1005, require a guide array to be transferred via Gateway cloning. This step outlines the construction of a Level 2 acceptor/Gateway entry clone that interfaces between these two systems. An easy way to achieve this is to amplify a Gateway cassette (a ccdB and a chloramphenicol-resistance gene) with added MoClo adapters, then TOPO ${ }^{\circledR}$ clone to pENTR.

1. Design and order the following primers:

a. F primer: CACCTGCCTTGGTCTCCATTAGGCACCCCAGGCTTT

b. R primer: TCCCTTGTCTTCGTCGACCTGCAGACTGGC

i. $\quad \mathrm{CACC}$ to direct the $\mathrm{TOPO}^{\circledR}$ cloning

ii. Overhangs to be used in Level 2 assembly

iii. Bpil recognition sequence

iv. Gateway cassette annealing sequence

Note: Check that primer annealing sequence matches yours and adjust if necessary. A cassette with chloramphenicol resistance and a ccdB gene comes out to about 1,500 bp (your variant could be different).

2. PCR amplify a fragment using the above primers. The template can be any vector with a Gateway cassette.

3. Verify a single strong band on a $1 \%$ agarose gel, then clean the remainder of the PCR product using a commercial DNA cleaning column kit.

4. Assemble the fragment to $\mathrm{pENTR}{ }^{\mathrm{TM}}$ in a $\mathrm{TOPO}^{\circledR}$ cloning reaction according to the manufacturer's instructions.

5. Transform $c c d B$ Survival ${ }^{\mathrm{TM}}$ cells with the assembly product and spread on plates containing kanamycin and chloramphenicol.

Alternative to Gateway cassette: The primer annealing sequences can be modified to amplify a LacZa cassette from Level 0 universal acceptor pAGM9121. In this case, transform typical TOP10 E. coli cells with the assembly product, plate to Blue/White screening plates with kanamycin, and screen white colonies.

C. Cloning procedure

1. Build Level 0 parts (Figure 1 step 1)

Note: Custom Level 0 parts require the MoClo Level 0 universal acceptor plasmid pAGM9121. This plasmid is available from Addgene either alone or as part of the MoClo Tool Kit (Reference 1). 
Step 1:

Build MoClo Level 0 parts

Step 2: Assemble Level 0 parts into a Level 1 acceptor to make a Level 1 guide gene

Step 3: Assemble up to 6 Level 1 genes to the custom Level 2/Gateway entry clone

Step 4: Transfer the guide array to the Expression vector in an $L R$ reaction
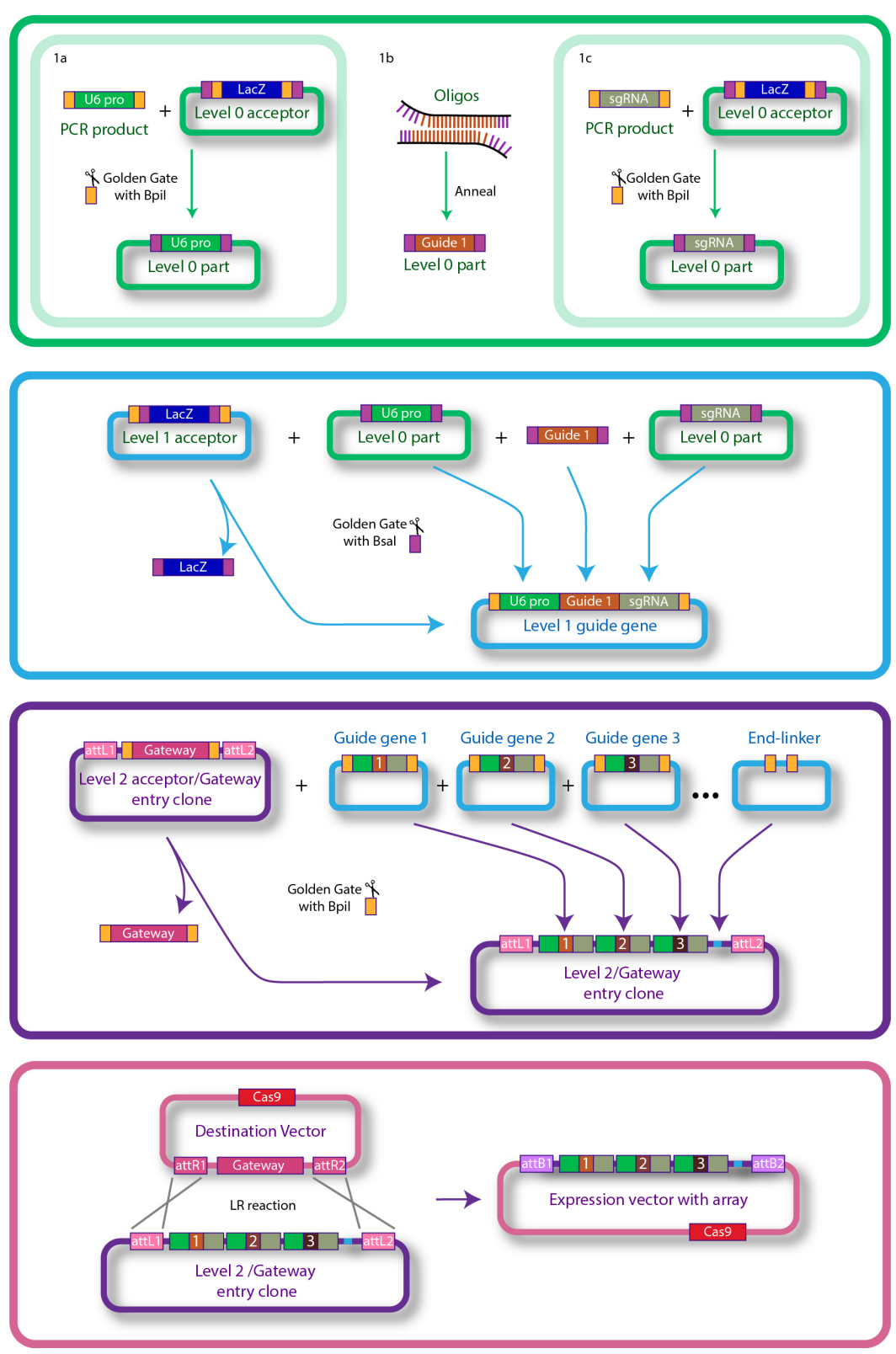

Figure 1. Four cloning steps to produce a guide array in an expression vector. Step 1: a)

PCR amplify the promoter(s), then clone them to the Level 0 universal acceptor in a Golden Gate reaction with Bpil. b) Order guide oligos and anneal them. c) PCR amplify the sgRNA scaffold, then clone it to the Level 0 universal acceptor in a Golden Gate reaction with Bpil. Step 2: For each Level 1 guide gene, assemble a promoter (in Level 0 universal vector), an annealed guide spacer, and the sgRNA scaffold (in Level 0 universal vector) to a Level 1 acceptor. This assembly takes place in a Golden Gate reaction using Bsal. Step 3: Up to six Level 1 guide genes, plus the appropriate end-linker, are assembled into the custom Level 2/Gateway entry clone. This assembly takes place in a Golden Gate reaction using Bpil. Step 4: The finished guide array is moved to the expression vector in an LR reaction.

a. Construct Level 0 promoter parts (Figure 1 step 1a) 
i. Design and order the following primers

Note: This example is for the ZmU6_Chr3 short promoter, see Table 1 for primer binding sequences for other promoters.

1) Forward primer: TGTGAAGACATCTCAGGAGCAAAAACATCCTCACAGGAAAG

2) Reverse primer: TGTGAAGACATCTCGGGTATGGTTCTTCCGCTCTG

Bpil recognition sequence

Overhang used in Level 0 assembly

Overhang used in Level 1 assembly

Promoter specific binding sequence

ii. Amplify promoter fragment in a PCR reaction with appropriate maize or rice genomic DNA as template. (Figure 2)

iii. Assemble promoter fragment into the Level 0 universal acceptor in a Golden Gate reaction using Bpil.

iv. Recover assembled constructs in TOP10 E. coli cells on plates containing spectinomycin.

v. Screen white colonies for correct construct by sequencing, colony PCR, or restriction fragment length analysis.

Note: To use more than one promoter type in the guide array, repeat in parallel the promoter primer design with the corresponding promoter binding sequence and assemble each PCR fragment to the same Level 0 universal acceptor. For transforming other species, any promoter sequence can be substituted here.

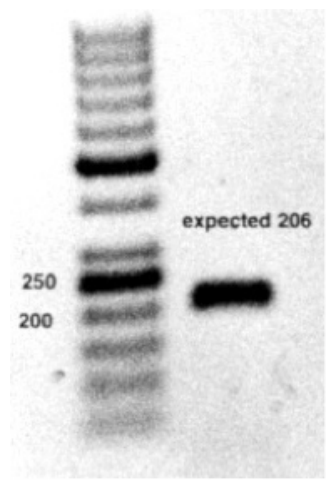

Figure 2. PCR product from Step C1a. The 206 bp promoter pZmU6-C3-Short was amplified from B73 genomic DNA using the above protocol and run on a $1 \%$ gel to ensure a strong single band at the expected size. If other bands are noticeable, it is advised to gel extract the correct size band, otherwise the entire PCR product can be cleaned and used without a size exclusion step.

b. Construct Level 0 guide spacer parts (Figure 1 step $1 \mathrm{~b}$ )

After choosing guide targets (see Procedure A), design oligos for each target as follows:

i. Start with the chosen 20 bp Cas9 guide target sequence, henceforth called (target) 
ii. To each (target), add the following extensions. The final oligo should be:

1) 5'-TGTGGTCTCATACC(target)GTTTTGAGACCACA-3'

Bsal recognition sequence

Overhangs used in Level 1 assembly

Note: The 5' extension overlap sequence TACC needs to match the last 4 bp of the promoter sequence, in this example the ZmU6_C3 short promoter (seen as GGTA in the promoter's Reverse primer). When using another promoter, modify the $5^{\prime}$ extension overhang to match the last $4 \mathrm{bp}$ of the promoter, i.e., TTTT for the pZmU6-C1 long promoter.

iii. Design another oligo that is the reverse complement of this $48 \mathrm{bp}$ oligo

iv. Order these oligos. When they arrive, prepare the fragment:

1) Rehydrate each oligo with ultrapure water to a concentration of $100 \mu \mathrm{M}$ in the stock tube.

2) To an empty thermocycler tube, add $10 \mu \mathrm{l}$ each of the two complimentary $100 \mu \mathrm{M}$ oligos, for a $20 \mu \mathrm{l}$ total reaction.

3) Anneal the oligos by running them on the following thermocycler program:

- $\quad 95^{\circ} \mathrm{C}$ for $2 \mathrm{~min}$

- Slowly decrease temperature at a rate of $\sim 2^{\circ} \mathrm{C}$ per min until $25^{\circ} \mathrm{C}$, or as slow as the thermocycler is capable

- Hold at $4{ }^{\circ} \mathrm{C}$

4) Store the annealed oligos at $-20^{\circ} \mathrm{C}$

c. Construct a Level 0 sgRNA part Figure 1 step 1c)

i. Order the following two oligos for use as primers in a PCR reaction:

1) TGTGAAGACATCTCAGTTTTAGAGCTAGAAATAGCAAG

2) TGTGAAGACATCTCGAGCGAAAAAAAGCACCGACTCGGTG

Bpil recognition sequence

Overhang used in Level 0 assembly

Overhang in Level 1 assembly

sgRNA specific binding sequence

ii. Using the above primers and a previous vector or transgenic organism containing the Streptococcus pyogenes sgRNA sequence, PCR amplify and check for a $116 \mathrm{bp}$ product on a $2 \%$ agarose gel.

iii. Assemble sgRNA fragment into the Level 0 universal acceptor in a Golden Gate reaction using Bpil.

iv. Recover assembled constructs in TOP10 E. coli cells on plates containing spectinomycin.

v. Screen white colonies for correct construct by sequencing, colony PCR, or restriction fragment length analysis.

2. Build Moclo Level 1 guide genes (Figure 1 step 2) 
Note: Guide genes are built by assembly into one of seven distinct Moclo Level 1 acceptor vectors. Each of these seven acceptors differs only by which position their gene will be assembled into a Level 2 acceptor. Each is produced in identical assembly protocols, so they can be constructed in parallel, followed by assembly into an array. Assemble the first guide gene in the Level 1 position 1 vector, the second guide gene to the Level 1 position 2 vector, and subsequent guide genes into subsequent position vectors.

a. Assemble the following Level 0 parts into a Level 1 acceptor vector in a Golden Gate reaction using Bsal.

i. Promoter in pAGM9121 (Level 0 part)

ii. Annealed guide oligos (Level 0 part)

iii. SgRNA in pAGM9121 (Level 0 part)

iv. Level 1 acceptor vector

b. Transform TOP10 E. coli cells with the assembly product and recover on plates containing carbenicillin.

c. Screen white colonies for correct construct by sequencing, colony PCR, or restriction fragment length analysis.

3. Assemble Level 1 guide genes to the Level 2 acceptor/Gateway entry clone (Figure 1 step 3) Note: The Level 2 assembly requires a Level 2 End-linker appropriate to your array size. To assemble more than six guides to the array, you must choose an End-linker that also contains a LacZ acceptor site such as pICH49300, which will allow subsequent reassemblies to add more guides. See the MoClo kit instructions for the appropriate End-linker constructs.

a. Assemble up to six Level 1 guide genes (and the appropriate End-linker) into the Level 2 acceptor/Gateway entry clone in a Golden Gate reaction using Bpil.

b. Recover assembled constructs in TOP10 E. coli cells on plates containing kanamycin. Do NOT recover in ccdB survival cells.

c. Screen colonies for correct construct by sequencing, colony PCR, or restriction fragment length analysis.

d. After screening colonies to find a correct assembly, it is a good idea to Sanger sequence the entire array at this time. If the array is too large to be sequenced from the backbone, design a sequencing primer that anneals to one of the internal guide spacers, rather than annealing to either the promoter or sgRNA scaffold, which will have multiple annealing sites that will prevent clean sequencing.

e. The finished guide array is now assembled in the Level 2/Gateway entry clone and is flanked by attL1 and attL2 sites.

Alternative: If your expression vector does not use Gateway recombination to accept the guides, instead assemble the Level 1 guide genes to a standard Level 2 acceptor such as pAGM4673 and then subclone the array by excision with DrallI.

4. Clone the guide array to the final expression vector (Figure 1 step 4) 
a. Set up a standard LR reaction according to the manufacturer's instructions with the two reagents:

i. Destination (expression) vector (such as pMCG1005) with attR1 and attR2 sites.

ii. The Level $2 /$ Gateway entry clone with guide array.

b. Recover the finished expression clone in TOP10 E. coli cells on plates containing spectinomycin (or whatever antibiotic resistance the expression vector has).

Note: Cas9 containing plasmids are notoriously difficult to work with in bacteria, possibly due to off-target editing in the bacterial genome. Word of mouth reports that incubation of cultures at $18{ }^{\circ} \mathrm{C}$ for two days instead of $37^{\circ} \mathrm{C}$ overnight will reduce the Cas 9 activity and facilitate easy cloning steps.

c. Validate the final construct with restriction digests and/or Sanger sequencing.

5. Transfer construct to Agrobacterium and verify stability

a. Transform Agrobacterium tumefaciens strain EHA101

i. Remove competent cells (generally $50-200 \mu \mathrm{l}$ ) from the $-80{ }^{\circ} \mathrm{C}$ freezer and thaw gradually on ice.

ii. When thawed, add $1 \mu \mathrm{l}$ of sequence validated expression clone miniprep and mix quickly by flicking.

iii. Place back on ice for 5 min.

iv. Place into a water bath at $37^{\circ} \mathrm{C}$ for $5 \mathrm{~min}$.

v. Remove from the water bath and place back on ice for $5 \mathrm{~min}$.

vi. Add $1 \mathrm{ml} \mathrm{SOC}$ media and place in a shaking incubator at $28^{\circ} \mathrm{C}$ for $90 \mathrm{~min}$.

vii. Plate to LB with rifampicin and the antibiotic to which the expression clone confers resistance and incubate for $48 \mathrm{~h}$ at $28^{\circ} \mathrm{C}$.

b. Pick 4 Agrobacterium colonies to screen. Because they are difficult to screen, and Cas9 plasmids are unstable, best practice is re-transform plasmid to $E$. coli to check that the correct vector has been recovered.

i. Grow a $5 \mathrm{ml}$ culture (LB + rifampicin + vector-specific antibiotic) of each Agrobacterium colony. Save $1 / 2 \mathrm{ml}$ from each and make a glycerol stock stored at $-80^{\circ} \mathrm{C}$.

ii. Miniprep the remaining $\sim 4.5 \mathrm{ml}$ of each with a commercial column kit. Yield will be low, this is ok.

iii. Heat shock transform $1 \mu$ of each miniprep to TOP10 E. coli cells, and plate to LB + vector-specific antibiotic.

iv. Pick three E. coli colonies per plate, culture, miniprep, and screen these by restriction digest.

v. If all three E. coli colonies from a single plate are correct according to the digest, the parent Agrobacterium colony is likely correct and ready to use for transforming plants. To make the final infection culture, use the glycerol stock that transformed the verified E. coli cells to transform maize callus tissue. 
D. General procedures used in this protocol

\section{PCR amplifications}

1. Use extracted genomic DNA diluted $50 \mathrm{ng} / \mu \mathrm{l}$ or miniprep plasmid DNA diluted to $1 \mathrm{ng} / \mu \mathrm{l}$ as template.

2. All oligo primers should be ordered with standard desalted purification, $25 \mathrm{nmol}$ range. Rehydrate to a concentration of $100 \mu \mathrm{M}$ in the stock tube, and make another tube with working dilution at $20 \mu \mathrm{M}$, store these both at $-20^{\circ} \mathrm{C}$.

3. Set up a $20 \mu \mathrm{l} P C R$ reaction on ice using a cloning-quality proofreading polymerase such as Phusion or Q5. This protocol will demonstrate with Phusion.

\begin{tabular}{|l|l|}
\hline Reagent & Quantity \\
\hline $5 x$ Phusion HF buffer & $4 \mu \mathrm{l}$ \\
\hline dNTPs mix $(10 \mathrm{mM})$ & $2 \mu \mathrm{l}$ \\
\hline Forward primer $(20 \mu \mathrm{M})$ & $0.5 \mu \mathrm{l}$ \\
\hline Reverse primer $(20 \mu \mathrm{M})$ & $0.5 \mu \mathrm{l}$ \\
\hline Template $(g D N A$ at $50 \mathrm{ng} / \mu \mathrm{l}$ or plasmid DNA at $1 \mathrm{ng} / \mu \mathrm{l})$ & $0.5 \mu \mathrm{l}$ \\
\hline Phusion polymerase & $0.2 \mu \mathrm{l}$ \\
\hline Water & $12.3 \mu \mathrm{l}$ (to $20 \mu \mathrm{l})$ \\
\hline
\end{tabular}

4. Run in a thermocycler with the following program:
a. Denature $98^{\circ} \mathrm{C} 1 \mathrm{~min}$
b. 25 cycles:
i. Denature $98^{\circ} \mathrm{C} 10 \mathrm{~s}$
ii. Anneal $68{ }^{\circ} \mathrm{C} 10 \mathrm{~s}$ (Adjust if necessary based on primers, or use a touchdown program)
iii. Extension $72^{\circ} \mathrm{C} 10 \mathrm{~s}$
c. Final Extension $72^{\circ} \mathrm{C} 1 \mathrm{~min}$
d. Hold at $4-10^{\circ} \mathrm{C}$

5. If using a plasmid template that contains the same antibiotic resistance gene as the vector you plan to clone to, you will need to first digest the PCR product to destroy template plasmid. Otherwise skip this step.
a. Add $0.5 \mu \mathrm{l}$ Dpnl to each PCR product, mix well by pipetting.
b. Incubate at $37^{\circ} \mathrm{C}$ for $15 \mathrm{~min}$.
c. Incubate at $80^{\circ} \mathrm{C}$ for $20 \mathrm{~min}$.

6. Check for fragment length and absence of other fragments by electrophoresis.
a. Load $1 \mu \mathrm{l} \mathrm{PCR}$ product into a $1 \%$ agarose gel. The expected amplicon size is $206 \mathrm{bp}$ for pZmU6-C3-Short (Figure 2).


b. If a single strong band is seen at the correct size, use a commercial DNA cleaning column kit to clean the rest of the PCR product.

c. If additional bands are present, run the remainder of the PCR product on a clean $1 \%$ gel, cut out the single expected band, and use a commercial gel extraction kit to recover the product.

7. Quantify the DNA using a NanoDrop or other similar spectrophotometer.

\section{Golden Gate assemblies}

1. Set up a Golden Gate reaction on ice (the NEBioCalculator tool is convenient for calculating moles of each reagent needed)

\begin{tabular}{|l|l|}
\hline Reagent & Quantity \\
\hline Acceptor vector (backbone) & $\sim 50 \mathrm{fmol}$ \\
\hline Inserts (each) & $\sim 100 \mathrm{fmol}$ \\
\hline 10x T4 Ligase buffer (with ATP) & $2 \mu \mathrm{l}$ \\
\hline T4 Ligase & $0.5 \mu \mathrm{l}$ \\
\hline Bpil or Bsal & $0.5 \mu \mathrm{l}$ \\
\hline Optional: BSA $1 \mathrm{mg} / \mathrm{ml}$ & $2 \mu \mathrm{l}$ \\
\hline Water & To $20 \mu \mathrm{l}$ \\
\hline
\end{tabular}

2. Assemble construct in a thermocycler with the following program:
a. 26 cycles:
$37^{\circ} \mathrm{C} 3 \mathrm{~min}$
$16{ }^{\circ} \mathrm{C} 4 \mathrm{~min}$
b. $50^{\circ} \mathrm{C} 5 \mathrm{~min}$
c. $80{ }^{\circ} \mathrm{C} 20 \mathrm{~min}$
d. Hold at $4-10{ }^{\circ} \mathrm{C}$, store at $-20^{\circ} \mathrm{C}$ long term

Note: If using a different promoter with an internal Bpil/Bsal cut site, omit the $50^{\circ} \mathrm{C}$ and $80^{\circ} \mathrm{C}$ steps.

3. Recover finished constructs by transformation of $E$. coli
a. Add $1 \mu \mathrm{l}$ assembly product to a tube of chemically competent $E$. coli cells such as TOP10 or DH5a, incubate on ice $30 \mathrm{~min}$, flicking tube gently every $10 \mathrm{~min}$ to mix.
b. Heat shock cells by placing in a $42{ }^{\circ} \mathrm{C}$ water bath for exactly $30 \mathrm{~s}$.
c. Immediately return tubes to ice and incubate for $2 \mathrm{~min}$.
d. Add to each tube $1 \mathrm{ml} \mathrm{SOC}$ media and place in a shaking incubator at $37^{\circ} \mathrm{C}$ for $1 \mathrm{~h}$.
e. Spin tubes on a tabletop centrifuge at 1,000 $\times g$ for $3 \mathrm{~min}$. 
f. Remove the majority of supernatant leaving the cell pellet and $\sim 100 \mu$ liquid. Using P200 pipette with sterile tip, very slowly and gently pipette up and down to resuspend the pellet.

g. Pipette $\sim 10 \mu \mathrm{l}$ to one blue/white screening plate with appropriate antibiotic and $\sim 90 \mu \mathrm{l}$ to another plate and immediately spread.

h. Incubate plates upside down at $37^{\circ} \mathrm{C}$ until colonies are average size, usually 16-24 h. Colonies may show blue color immediately, but the color is usually faint. To accentuate color, refrigerate plate for another day or two, which will inhibit further colony growth but allow the color to develop more.

4. Screen for correct construct

a. Screen white colonies for correct construct by sequencing, colony PCR, or restriction fragment length analysis (hint, use Bpil/Bsal to check insert size directly, when applicable). Including a blue colony as a negative control is a good idea because it will not contain the expected insert.

b. Save verified construct as a glycerol stock ( $20 \%$ glycerol) at $-80^{\circ} \mathrm{C}$.

5. Prepare a miniprep of one verified colony and quantify DNA mass with a spectrophotometer.

\section{Acknowledgments}

The authors acknowledge the NSF PGRP program grant \#1546837 for supporting this work. Thanks also to the members of the Jackson and Lippman labs at Cold Spring Harbor Labs: Edgar Demesa Arevalo, Hannes Claeys, Lei Liu, Tara Skopelitis, and Byoung II Je, and Choon-Tak Kwon, for providing guidance and promoter sequence definitions.

Competing interests: The authors declare no competing financial interest.

\section{References}

1. Addgene: MoClo Toolkit [Internet]. [cited 19 Dec 2018]. Available: https://www.addgene.org/cloning/moclo/marillonnet/.

2. Char, S. N., Neelakandan, A. K., Nahampun, H., Frame, B., Main, M., Spalding, M. H., Becraft, P. W., Meyers, B. C., Walbot, V., Wang, K. and Yang, B. (2017). An Agrobacterium-delivered CRISPR/Cas9 system for high-frequency targeted mutagenesis in maize. Plant Biotechnol J 15(2): 257-268.

3. Feng, C., Yuan, J., Wang, R., Liu, Y., Birchler, J. A. and Han, F. (2016). Efficient targeted genome modification in maize using CRISPR/Cas9 system. J Genet Genomics 43(1): 37-43.

4. Haeussler, M., Schonig, K., Eckert, H., Eschstruth, A., Mianne, J., Renaud, J. B., Schneider-Maunoury, S., Shkumatava, A., Teboul, L., Kent, J., Joly, J. S. and Concordet, J. P. (2016). Evaluation of off-target and on-target scoring algorithms and integration into the guide RNA selection tool CRISPOR. Genome Biol 17(1): 148. 
5. Lee, K., Zhang, Y., Kleinstiver, B. P., Guo, J. A., Aryee, M. J., Miller, J., Malzahn, A., Zarecor, S., Lawrence-Dill, C. J., Joung, J. K., Qi, Y. and Wang, K. (2019). Activities and specificities of CRISPR/Cas9 and Cas12a nucleases for targeted mutagenesis in maize. Plant Biotechnol J 17(2): 362-372.

6. Qi, X., Dong, L., Liu, C., Mao, L., Liu, F., Zhang, X., Cheng, B. and Xie, C. (2018). Systematic identification of endogenous RNA polymerase III promoters for efficient RNA quide-based genome editing technologies in maize. Crop J 6: 314-320.

7. Rodríguez-Leal, D., Lemmon, Z. H., Man, J., Bartlett, M. E. and Lippman, Z. B. (2017). Engineering quantitative trait variation for crop improvement by genome editing. Cell 171(2): 470-480 e8.

8. Svitashev, S., Young, J. K., Schwartz, C., Gao, H., Falco, S. C. and Cigan, A. M. (2015). Targeted Mutagenesis, Precise Gene Editing, and Site-Specific Gene Insertion in Maize Using Cas9 and Guide RNA. Plant Physiol 169(2): 931-945.

9. Vad-Nielsen, J., Lin, L., Bolund, L., Nielsen, A. L. and Luo, Y. (2016). Golden Gate Assembly of CRISPR gRNA expression array for simultaneously targeting multiple genes. Cell Mol Life Sci 73(22): 4315-4325.

10. Werner, S., Engler, C., Weber, E., Gruetzner, R. and Marillonnet, S. (2012). Fast track assembly of multigene constructs using Golden Gate cloning and the MoClo system. Bioeng Bugs 3(1): 38-43. 